\title{
Variational Iteration Method for Logistic Equation with Piecewise Constant Arguments
}

\author{
Qi Wang* and Ziting Liu \\ School of Applied Mathematics, Guangdong University of Technology, Guangzhou, Guangdong 510006, China \\ ${ }^{*}$ Corresponding author
}

\begin{abstract}
In this paper, variational iteration method is applied for finding the analytic approximate solution of Logistic equation with piecewise constant arguments. A correction functional is constructed by a general Lagrange multiplier, which can be identified by variational theory. Moreover, the iteration formula is obtained. The flexibility and adaptation provided by this method have been verified by an intuitive numerical example.
\end{abstract}

Keywords—variational iteration method; Logistic equation; approximate solution

\section{INTRODUCTION}

In recent years, differential equations with piecewise constant arguments (EPCA) have attracted great deal of attention of researchers in mathematical and some other fields in science. EPCA are useful to describe certain actual phenomena from physics, biology, control science and the other scientific fields [1-4]. Some analytical results for EPCA have been presented by many authors (see [5-7]). A detailed introduction to the theory of EPCA can be found in Wiener's monograph [8].

Generally speaking, it is difficult or even impossible to obtain an exact solution of EPCA. Hence, in recent years, efforts have been made to develop various numerical methods to solve them and study the corresponding algorithmic theory. Liu et al. [9] first investigated the numerical stability of the Runge-Kutta methods for the most basic EPCA. Next, numerical treatment of EPCA has developed rapidly. The oscillation of numerical solution in the $\theta$-method and the Runge-Kutta methods for a linear retarded type EPCA was considered in [10, 11], respectively. Wang and Li [12] analyzed the numerical dissipativity of neutral EPCA, some sufficient conditions for the dissipativity of the equation were given. In [13], asymptotical stability of Runge-Kutta methods for the advanced linear impulsive EPCA was studied. As far as authors' knowledge goes, only few results are presented in the case of analytical approximate treatment of EPCA. In this paper we will study the problem of how to get the approximations of Logistic EPCA by use of variational iteration method.

The variational iteration method (VIM) [14-17], which is a well-established technique with wide applications for ordinary differential equations, partial differential equations and delay differential equations, etc. More materials of the classical solution techniques that are most commonly used to solve equations and systems, such as the Adomian decomposition method (ADM) [18], Differential transformation method (DTM) [19], Homotopy analysis method (HAM) [20] and the Homotopy perturbation method (HPM) [21]. The VIM is the most effective and convenient one for both weakly and strongly nonlinear equations. This method has been shown to effectively, easily, and accurately solve a large class of linear and nonlinear problems.

In this paper we consider a Logistic EPCA

$$
u^{\prime}(t)=a u(t)(r-b u([t])), \quad u(0)=u_{0},
$$

where $a, b>0, r, u_{0} \in R$ and [.] denotes the greatest integer function. The main purpose of this paper is to extend the VIM method to find the approximate solution of (1).

\section{PRELIMINARIES}

Definition 1[8] A solution of (1) on $[0, \infty)$ is a function $u(t)$ that satisfies the conditions:

(i) $u(t)$ is continuous on $[0, \infty)$,

(ii) The derivative $u^{\prime}(t)$ exists at each point $t \in[0, \infty)$, with the possible exception of the point $t=n, n=0,1, \cdots$, where one-sided derivatives exist,

(iii) Equation (1) is satisfied on each interval $[n, n+1)$ $\subset[0, \infty)$.

Next, we will introduce the idea of VIM. The VIM is the general Lagrange method, in which an extremely accurate approximation at some special point can be obtained, but not an analytical solution. To illustrate the basic idea of the VIM we consider the following general differential equation

$$
L u+N u=g(x),
$$

where $L$ and $N$ are linear and nonlinear operator, respectively, and $g(x)$ is the inhomogeneous term. Moreover, the author proposed the VIM where a correction functional for (2) can be written as 


$$
u_{n+1}(x)=u_{n}(x)+\int_{0}^{x} \lambda(t)\left[L u_{n}(t)+N \tilde{u}_{n}(t)-g(t)\right] d t,
$$

where $\lambda$ is a general Lagrange's multiplier, which can be identified optimally via the variational theory, and $\tilde{\boldsymbol{u}}_{\boldsymbol{n}}$ as a restricted variation which means $\boldsymbol{\delta} \tilde{\boldsymbol{u}}_{\boldsymbol{n}}=\mathbf{0}$. It is to be noted that the Lagrange multiplier $\lambda$ can be a constant or a function. The VIM should be employed by following two main steps. It is required first to determine the Lagrange multiplier $\lambda$ that can be identified optimally via integration by parts and by using a restricted variation. Having $\lambda$ determined, an iteration formula, without restricted variation, should be used for the determination of the successive approximations $u_{n+1}(x)$ of the solution $u(x)$. The zeroth approximation $u_{0}$ can be any selective function. Consequently, the solution is given by

$$
u(x)=\lim _{n \rightarrow \infty} u_{n}(x)
$$

\section{APPLICATIONS}

Now, we apply the VIM to (1). A correction functional is constructed as follows

$u_{n+1}(t)=u_{n}(t)+\int_{0}^{t} \lambda(s)\left[u_{n}^{\prime}(s)-\operatorname{ar} \tilde{u}_{n}(s)+a b \tilde{u}_{n}(s) \tilde{u}_{n}([s])\right] d s$

Taking the variation of both sides of (5) with respect to the independent variable $u_{n}$

$$
\begin{aligned}
\delta u_{n+1}(t) & =\delta u_{n}(t)+\delta \int_{0}^{t} \lambda(s) u_{n}^{\prime}(s) d s, \\
& =\delta u_{n}(t)+\lambda(t) \delta u_{n}(t)-\int_{0}^{t} \lambda^{\prime}(s) \delta u_{n}(s) d s, \\
& =(1+\lambda(t)) \delta u_{n}(t)-\int_{0}^{t} \lambda^{\prime}(s) \delta u_{n}(s) d s,
\end{aligned}
$$

which yields the stationary conditions

$$
\left\{\begin{array}{l}
1+\lambda(t)=0 \\
\left.\lambda^{\prime}(s)\right|_{s=t}=0
\end{array}\right.
$$

This in turn gives $\lambda(s)=-1$. Substituting this value of the Lagrange multiplier into the functional (5) gives the iteration formula

$$
\begin{aligned}
u_{n+1}(t)= & u_{n}(t) \\
& -\int_{0}^{t}\left[u_{n}^{\prime}(s)-\operatorname{aru}_{n}(s)+a b u_{n}(s) u_{n}([s])\right] d s
\end{aligned}
$$

We select the initial value $u_{0}$ as the zeroth approximation, so we obtain the following successive approximations

$$
\begin{aligned}
& u_{0}(t)=u_{0} \\
& u_{1}(t)=u_{0}(t)-\int_{0}^{t}\left[u_{0}^{\prime}(s)-a r u_{0}(s)+a b u_{0}(s) u_{0}([s])\right] d s \\
& u_{2}(t)=u_{1}(t)-\int_{0}^{t}\left[u_{1}^{\prime}(s)-a r u_{1}(s)+a b u_{1}(s) u_{1}([s])\right] d s
\end{aligned}
$$

$$
u(t)=\lim _{n \rightarrow \infty} u_{n}(t)
$$

In order to overcome the difficulty from the greatest integer function [.], we can consider the above iteration formula in a series of intervals: $[0,1),[1,2),[2,3), \ldots,[n, n+1)$, where $n=0,1, \cdots$. Following this way, each integral in iteration formulas will be easily computed.

The above analysis yields the following theorem.

Theorem 1 The VIM solution of (1) can be determined by (8) with the iteration (7).

In the next section, the VIM is successfully applied for solving a nonlinear EPCA.

\section{ONE EXAMPLE}

Let $a=b=2, r=u_{0}=1$ in (1), from (7) we have

$$
\begin{aligned}
u_{n+1}(t)= & u_{n}(t) \\
& -\int_{0}^{t}\left[u_{n}^{\prime}(s)-2 u_{n}(s)+4 u_{n}(s) u_{n}([s])\right] d s,
\end{aligned}
$$

thus, when $t \in[0,1)$

$$
\begin{aligned}
u_{1,0}(t)= & 1 \\
u_{1,1}(t)= & u_{1,0}(t) \\
& \quad-\int_{0}^{t}\left[u_{1,0}^{\prime}(s)-2 u_{1,0}(s)+4 u_{1,0}(s) u_{1,0}([s])\right] d s \\
= & 1-2 t
\end{aligned}
$$




$$
\begin{aligned}
u_{1,2}(t)= & u_{1,1}(t) \\
& -\int_{0}^{t}\left[u_{1,1}^{\prime}(s)-2 u_{1,1}(s)+4 u_{1,1}(s) u_{1,1}([s])\right] d s \\
= & 1-2 t+2 t^{2}, \\
u_{1,3}(t)= & u_{1,2}(t) \\
& -\int_{0}^{t}\left[u_{1,2}^{\prime}(s)-2 u_{1,2}(s)+4 u_{1,2}(s) u_{1,2}([s])\right] d s \\
= & 1-2 t+2 t^{2}-\frac{4}{3} t^{3}, \\
u_{1,4}(t)= & u_{1,3}(t) \\
& -\int_{0}^{t}\left[u_{1,3}^{\prime}(s)-2 u_{1,3}(s)+4 u_{1,3}(s) u_{1,3}([s])\right] d s \\
= & 1-2 t+2 t^{2}-\frac{4}{3} t^{3}+\frac{2}{3} t^{4},
\end{aligned}
$$

When $t \in[1,2)$

$$
\begin{aligned}
u_{2,0}(t)= & 1, \\
u_{2,1}(t)= & u_{2,0}(t) \\
& -\int_{0}^{t}\left[u_{2,0}^{\prime}(s)-2 u_{2,0}(s)+4 u_{2,0}(s) u_{2,0}([s])\right] d s \\
= & 1-2 t, \\
u_{2,2}(t)= & u_{2,1}(t) \\
& -\int_{0}^{t}\left[u_{2,1}^{\prime}(s)-2 u_{2,1}(s)+4 u_{2,1}(s) u_{2,1}([s])\right] d s \\
= & 1+6 t-6 t^{2}, \\
u_{2,3}(t)= & u_{2,2}(t) \\
& \quad-\int_{0}^{t}\left[u_{2,2}^{\prime}(s)-2 u_{2,2}(s)+4 u_{2,2}(s) u_{2,2}([s])\right] d s \\
= & 1-2 t-6 t^{2}+4 t^{3}, \\
& \\
u_{2,4}(t)= & u_{2,3}(t) \\
& \quad \int_{0}^{t}\left[u_{2,3}^{\prime}(s)-2 u_{2,3}(s)+4 u_{2,3}(s) u_{2,3}([s])\right] d s \\
= & 1+14 t-14 t^{2}-28 t^{3}+14 t^{4},
\end{aligned}
$$

When $t \in[2,3)$

$$
\begin{aligned}
& u_{3,0}(t)=1 \text {, } \\
& u_{3,1}(t)=u_{3,0}(t) \\
& -\int_{0}^{t}\left[u_{3,0}^{\prime}(s)-2 u_{3,0}(s)+4 u_{3,0}(s) u_{3,0}([s])\right] d s \\
& =1-2 t \text {, } \\
& u_{3,2}(t)=u_{3,1}(t) \\
& -\int_{0}^{t}\left[u_{3,1}^{\prime}(s)-2 u_{3,1}(s)+4 u_{3,1}(s) u_{3,1}([s])\right] d s \\
& =1+14 t-14 t^{2} \text {, } \\
& u_{3,3}(t)=u_{3,2}(t) \\
& -\int_{0}^{t}\left[u_{3,2}^{\prime}(s)-2 u_{3,2}(s)+4 u_{3,2}(s) u_{3,2}([s])\right] d s \\
& =1+110 t+770 t^{2}-\frac{1540}{3} t^{3}, \\
& u_{3,4}(t)=u_{3,3}(t) \\
& -\int_{0}^{t}\left[u_{3,3}^{\prime}(s)-2 u_{3,3}(s)+4 u_{3,3}(s) u_{3,3}([s])\right] d s, \\
& =1+\frac{9674}{3} t+\frac{532070}{3} t^{2}+\frac{7448980}{9} t^{3}-\frac{3724490}{9} t^{4} \text {. }
\end{aligned}
$$

In a word, in the interval $[n, n+1)$, we have the following iteration formulas:

$$
\begin{aligned}
& u_{n+1,0}(t)=1, \\
& u_{n+1, m}(t)=u_{n+1, m-1}(t) \\
& -\int_{0}^{t}\left[u_{n+1, m-1}^{\prime}(s)-2 u_{n+1, m-1}(s)+4 u_{n+1, m-1}(s) u_{n+1, m-1}([s])\right) d s .
\end{aligned}
$$

In view of (8), we can obtain the approximate analytical solution. Usually, the $m+1$ th approximation is used for numerical purposes.

In Table 1 we compare the absolute errors (AE) of the VIM ( $m=5,6$ ) with the ones of the $\theta$-method [22] with $\theta=0.6$ and step size $h=0.02$. From this table we can easily see that the VIM method has good convergence even though with the lower times of iterations. It is obvious that this method gives rapid convergent successive approximations without any restrictive assumptions or transformation that may change the physical behavior of the problem. 
TABLE I. COMPARISON OF THE AE

\begin{tabular}{|l|l|l|l|}
\hline \multirow{2}{*}{$t$} & \multicolumn{2}{|c|}{ VIM } & \multirow{2}{*}{$\theta$-method } \\
\cline { 2 - 3 } & $m=5$ & $m=6$ & \\
\hline 0.5 & $2.05 \mathrm{E}-6$ & $6.37 \mathrm{E}-7$ & $3.53 \mathrm{E}-4$ \\
\hline 1.0 & $4.24 \mathrm{E}-6$ & $7.79 \mathrm{E}-7$ & $3.16 \mathrm{E}-4$ \\
\hline 1.5 & $6.16 \mathrm{E}-6$ & $5.57 \mathrm{E}-6$ & $4.81 \mathrm{E}-3$ \\
\hline 2.0 & $5.24 \mathrm{E}-5$ & $6.35 \mathrm{E}-6$ & $4.62 \mathrm{E}-3$ \\
\hline 2.5 & $5.41 \mathrm{E}-5$ & $7.75 \mathrm{E}-6$ & $8.65 \mathrm{E}-3$ \\
\hline 3.0 & $7.56 \mathrm{E}-5$ & $2.08 \mathrm{E}-5$ & $3.96 \mathrm{E}-2$ \\
\hline 3.5 & $4.84 \mathrm{E}-4$ & $3.92 \mathrm{E}-5$ & $4.37 \mathrm{E}-2$ \\
\hline 4.0 & $8.36 \mathrm{E}-4$ & $7.52 \mathrm{E}-5$ & $4.93 \mathrm{E}-2$ \\
\hline
\end{tabular}

\section{ACKNOWLEDGMENT}

This work is supported by the Natural Science Foundation of Guangdong Province (No. 2017A030313031).

\section{REFERENCES}

[1] D. Altintan, Extension of the logistic equation with piecewise constant arguments and population dynamics, M.Sc.Thesis, Middle East Technical University, 2006

[2] K.L. Cooke, J. Weiner, A survey of differential equation with piecewise continuous argument, Lecture Notes in Mathematics, Springer, Berlin, 1991.

[3] L. Dai, L. Fan, “Analytical and numerical approaches to characteristics of linear and nonlinear vibratory systems under piecewise discontinuous disturbances,” Commun. Nonlinear Sci. Numer. Simul., vol. 9, pp. 417429, 2004.

[4] F. Gurcan, F. Bozkurt, "Global stability in a population model with piecewise constant arguments,” J. Math. Anal. Appl., vol. 360, pp. 334342, 2009.

[5] K.L. Cooke, J. Wiener, "Retarded differential equations with piecewise constant delays,” J. Math. Anal. Appl., vol. 99, pp. 265-297, 1984.

[6] K.L. Cooke, J. Wiener, "Stability regions for linear equations with piecewise continuous delay,” Comput. Math. Appl., vol. 12, pp. 695701, 1986 .

[7] H. Bereketoglu, G. Seyhan, and A. Ogun, "Advanced impulsive differential equations with piecewise constant arguments,” Math. Model. Anal., vol. 15, pp. 175-187, 2010 .

[8] J. Wiener, Generalized Solutions of Differential Equations, World Scientific, Singapore, 1993.

[9] M.Z. Liu, M.H. Song, and Z.W. Yang, "Stability of Runge-Kutta methods in the numerical solution of equation $u^{\prime}(t)=a u(t)+a_{0} u([t])$," J. Comput. Appl. Math., vol. 166, pp. 361-370, 2004.

[10] M.Z. Liu, J.F. Gao, and Z.W. Yang, “Oscillation analysis of numerical solution in the $\theta$-methods for equation $x^{\prime}(t)+a x(t)+a_{1} x([t-1])=0$," Appl. Math. Comput., vol. 186, pp. 566-578, 2007.

[11] M.Z. Liu, J.F. Gao, and Z.W. Yang, "Preservation of oscillations of the Runge-Kutta method for equation $x^{\prime}(t)+a x(t)+a_{1} x([t-1])=0$," Comput. Math. Appl., vol. 58, pp. 1113-1125, 2009.

[12] W.S. Wang, S.F. Li, "Dissipativity of Runge-Kutta methods for neutral delay differential equations with piecewise constant delay,” Appl. Math. Lett., vol. 21, pp. 983-991, 2008.

[13] G.L. Zhang, M.H. Song, “Asymptotical stability of Runge-Kutta methods for advanced linear impulsive differential equations with piecewise constant arguments,” Appl. Math. Comput., vol. 259, pp. 831837, 2015.

[14] A.M. Wazwaz, "The Variational iteration method for solving new fourth-order Emden-Fowler type equations,” J. Math. Chem., vol.55, pp. 799-817, 2017.

[15] J.H. He, "Variational iteration method-Some recent results and new interpretations,” J. Comput. Appl. Math., vol. 207, pp. 3-17, 2007.

[16] J.H. He, X.H. Wu, "Variational iteration method: New development and applications,” Comput. Math. Appl., vol. 54, pp. 881-894, 2007.
[17] J.H. He, G.C. Wu, and F. Austin, "The variational iteration method which should be followed,” Nonlinear Sci. Lett. A, vol. 1, pp. 1-30, 2010.

[18] M.M. Al-Sawalha, M.S.M. Noorani, and I. Hashim, "On accuracy of Adomian decompositionmethod for hyperchaotic Rössler system," Chaos Solitons Fractals., vol. 40, pp. 1801-1807, 2009.

[19] M.M. Al-Sawalha, M.S.M. Noorani, and I. Hashim, “Application of the differential transformation method for the solution of the hyperchaotic Rössler system,” Comm. Non. Sci. Num. Simu., vol. 14, pp. 1509-1514, 2009.

[20] F.M. Allan, "Construction of analytic solution to chaotic dynamical systems using the homotopy analysis method," Chaos Solitons Fractals, vol. 39, pp. 1744-1752, 2009.

[21] J.H. He, "Homotopy perturbation method for bifurcation of nonlinear problems,” Int. J. Nonlinear Sci. Numer. Simu., vol. 6, pp. 207-208, 2005.

[22] M.H. Song, Z.W. Yang, and M.Z. Liu, "Stability of $\theta$-methods for advanced differential equations with piecewise continuous arguments," Comput. Math. Appl., vol. 49, pp. 1295-1301, 2005. 